\title{
LETTERS
}

\section{Unmasked tuberculosis or lymphoma in late AIDS presenters: a difficult differential diagnosis}

\section{To the Editors:}

Despite dramatic progress in the management of HIV infection [1], late presentation remains a major threat to survival [2]. Difficult diagnosis of AIDS-related illnesses could pose additional challenge. Tuberculosis (TB) is considered to be the most frequent opportunistic infection worldwide. Smearnegative pulmonary TB or extrapulmonary TB diagnosis may be difficult to rule out, either because of inadequate laboratory markers, or overlapping clinical presentation with other conditions, such as lymphoproliferative disorders. In addition, in the era of combination antiretroviral therapy (cART), unmasked TB could develop as immune reconstitution inflammatory syndrome (IRIS) after cART is commenced in treatment-naïve subjects with advanced HIV infection. Here we describe two cases of AIDS-related diseases, with similar presentation but different diagnosis and outcome.

The first patient was a 37-yr-old male, who was born in Togo and had lived in Italy for 2 yrs, presented to our attention in May 2008 for mild fever and chest pain. He had been diagnosed for the first time with pulmonary TB (acid-fast bacilli in the sputum smear) and advanced HIV infection (CD4+ T-cell count 40 cells $\cdot \mathrm{mm}^{-3}$ ) 2 months earlier, elsewhere. The patient had been treated for 2 months with rifampin, isoniazid, ethambutol and pyrazinamide, but had not started cART. On admission, physical examination showed enlargement of the liver, but no superficial lymphadenopathy. Laboratory tests showed mild anaemia (haemoglobin $11.7 \mathrm{~g} \cdot \mathrm{dL}^{-1}$, mean corpuscolar volume $75.9 \mathrm{fL}$ ), normal white blood cells and platelet count, and increased erythrocyte sedimentation rate $\left(72 \mathrm{~mm} \cdot \mathrm{h}^{-1}\right)$ and levels of serum lactate dehydrogenase $(\mathrm{LDH}) \quad\left(1,173 \mathrm{IU} \cdot \mathrm{L}^{-1}\right)$. Chest radiography revealed bilateral perihilar and lower right lung infiltrates. Sputum smear did not reveal the presence of acid-fast bacilli. A computed tomography (CT) scan of chest and abdomen showed an abdominal mass of $10 \times 6 \mathrm{~cm}$ in the pelvis and thickening of ileum wall, while nuclear magnetic resonance of the brain was normal. These findings were considered indicative of abdominal TB and a transcutaneous biopsy was not performed because of the risk of intestinal perforation. Mycobacterium antibiogram was not available at that time; therefore, a multidrug resistant TB could not be excluded. Moreover, a paradoxical worsening of clinical conditions even in the absence of cART has been previously reported as a form of IRIS, which was, therefore, considered in the differential diagnosis. Corticosteroid treatment was not prescribed owing to the uncertainty of diagnosis in the current stable patient's conditions. After 2 weeks, clinical conditions progressively deteriorated: haemoglobin dropped to $8 \mathrm{~g} \cdot \mathrm{dL}^{-1}, \mathrm{LDH}$ increased to $3,126 \mathrm{IU} \cdot \mathrm{L}^{-1}$, pulmonary infiltrates expanded, breathing rate increased to 40 breaths $\cdot \mathrm{min}^{-1}$, compensating arterial hypoxaemia. Pending bronchoscopy results, empirical therapy with cotrimoxazole against suspected pulmonary pneumocystosis and large-spectrum antibiotic treatment with piperacilline/ tazobactam did not lead to clinical improvement. Sputum, urine and stool smears were negative for acid-fast bacilli, thus supporting the hypothesis of IRIS. TB susceptibility test of the originally isolated strain became available, showing full sensitivity to the ongoing therapy, which was therefore confirmed. Thereafter, the patient developed left eye exophthalmos, and CT scan revealed a retro-orbitary mass. A transnasal biopsy led to the diagnosis of B-cell lymphoma. Bone marrow biopsy was performed for staging, and characterised the malignancy as a Burkitt's lymphoma. Unfortunately, diagnosis arrived too late; the patient died of septic shock, possibly secondary to intestinal perforation.

The second patient was a 59-yr-old, HIV-infected male, born in Angola and living in Italy, who had started first-line cART with zidovudine, lamivudine and nevirapine 6 months earlier (nadir CD4+ T-cell count 21 cells $\cdot \mathrm{mm}^{-3}$ ). He presented with fever $\left(39.5^{\circ} \mathrm{C}\right)$, weight loss and pancytopenia. At that time, his CD4+ T-cell count was 65 cells $\cdot \mathrm{mm}^{-3}$ and viral load was undetectable. Complete blood cell count showed: white blood cells 210 cells $\cdot \mathrm{mm}^{-3}$, red blood cells $1,620,000$ cells $\cdot \mathrm{mm}^{-3}$, haemoglobin $5.1 \mathrm{~g} \cdot \mathrm{dL}^{-1}$ and platelets $104,000 \mathrm{cells} \cdot \mathrm{mm}^{-3}$. The severe and rapid-onset pancytopenia led diagnostic suspicion towards haematological malignancies, and a bone marrow biopsy revealed a monocyte-macrophage activation syndrome, strongly supporting the suspect of lymphoproliferative disease. Chest radiograph showed micronodular infiltrates. Repeated sputum, bronchoalveolar lavage, urine and peripheral blood examinations were negative, while only a direct stool smear test revealed few acid-fast bacilli. Although the clinical TB presentation was actually atypical, anti-mycobacterial treatment with rifampin, isoniazid, ethambutol and pyrazinamide was started and progressively led to clinical amelioration, with rapid resolution of pancytopenia. Eventually, after 8 weeks of incubation, Mycobacterium tuberculosis was isolated from blood cultures of bone marrow aspirate, confirming the diagnosis of unmasked TB presentation

Late presentation of advanced HIV infection still has high mortality, which is driven either by the severity of AIDS-related disease or by the consequences of IRIS and unmasked TB. In this context, timely cART initiation becomes a critical issue.

In this report, both patients originated from African countries with high TB prevalence, and both presented with advanced HIV infection, haematological abnormalities and underlying mycobacterial disease. 
In the first case, the fear of IRIS led to a delay in cART initiation. Despite the fact that IRIS can lead to a paradoxical exacerbation of the clinical status, a diagnosis of TB should not prevent cART introduction in patients with advanced HIV infection. Previous studies have suggested that simultaneous use of CART and anti-TB treatment in patients with CD4+ Tcell counts $<200$ cells $\cdot \mathrm{mm}^{-3}$ could have a significant impact on survival [3, 4]. Moreover, a recent randomised trial demonstrated that, at least in resource-limited settings, cART also has the potential to reduce mortality in patients with relatively conserved immune function (i.e. CD4+ T-cell counts 200500 cells $\cdot \mathrm{mm}^{-3}$ ) [5].

Worsening of the patient's symptoms were initially attributed to TB progression or to IRIS, which can sometimes occur after specific treatment initiation, even in the absence of antiretroviral therapy [6]. Nonetheless, concomitant opportunistic infections are likely in HIV-infected patients, particularly when CD4+ T-cell count is low, and should always be ruled out when anti-mycobacterial treatment fails to improve the patient's conditions. In these circumstances, a more rapid and aggressive diagnostic work-up could have substantially improved the patient's outcome.

While in the first patient the symptoms were not immediately suggestive of lymphoma because of the concurrent pulmonary $\mathrm{TB}$, in the second patient, who presented with a more evident haematological impairment, the haemophagocytic syndrome was probably an expression of IRIS, that unmasked disseminated TB [7]. It is plausible that concurrent TB and HIV infection contributed to the development of haemophagocytic syndrome, whose mortality rate greatly depends on timely diagnosis and treatment of the underlying condition [8].

In the western world HIV mortality has dramatically decreased with the introduction of cART, but HIV patients still die when late HIV diagnosis combines with severe opportunistic diseases or HIV-related malignancies. Globalisation and migration from poor-income to high-income countries have contributed to raising the latent and overt $\mathrm{TB}$ burden in industrialised countries, resulting in the need for improving disease control strategies. Thus, a special effort, particularly in patients with advanced HIV infection who are coming from endemic TB areas, such as sub-Saharan African countries, should be made to screen for underlying TB, in order to prevent severe unmasking forms of the disease. Therefore, we would suggest that acid-fast bacilli are thoroughly sought for in biological fluids such as sputum, blood, urine and stools in patients with CD4+ T cell counts $<200$ cells $\cdot \mathrm{mm}^{-3}$. Moreover, new tools in TB diagnostics, such as molecular biology essays (PCR and in situ hybridisation by specific probe) could offer a great advantage in this task, but there is still a need of a novel rapid diagnostic technology which allows to discriminate between latent and disseminated active TB infections [9].

These two case reports represent two unusual presentation of AIDS-defining illnesses in which the complex interplay and mutual masking between an underlying opportunistic infection and host immune-haematological response could render the differential diagnosis a critical task for the clinician. Aggressive diagnostic and treatment efforts should be engaged to achieve possible recovery of these severe syndromes [10].

\section{A. Soria, S. Foresti, P. Fortuna, A. Dolara, A. Bandera, G. Lapadula and A. Gori}

Division of Infectious Diseases, Dept of Internal Medicine, San Gerardo Hospital, University of Milano-Bicocca, Monza, Italy.

Correspondence: A. Soria, Division of Infectious Diseases, Dept of Internal Medicine, San Gerardo Hospital, University of Milano-Bicocca, Via Pergolesi 33, 20052 Monza (MI), Italy. E-mail: a.soria@hsgerardo.org

Statement of Interest: None declared.

\section{REFERENCES}

1 Palella FJ Jr, Delaney KM, Moorman AC, et al. Declining morbidity and mortality among patients with advanced human immunodeficiency virus infection. HIV Outpatient Study Investigators. N Engl J Med 1998; 338: 853-860.

2 Sabin CA, Smith CJ, Youle M, et al. Deaths in the era of HAART: contribution of late presentation, treatment exposure, resistance and abnormal laboratory markers. AIDS 2006; 20: 67-71.

3 Schiffer JT, Sterling TR. Timing of antiretroviral therapy initiation in tuberculosis patients with AIDS: a decision analysis. J Acquir Immune Defic Syndr 2007; 44: 229-234.

4 Velasco M, Castilla V, Sanz J, et al. Effect of simultaneous use of highly active antiretroviral therapy on survival of HIV patients with tuberculosis. J Acquir Immune Defic Syndr 2009; 50: 148-152.

5 Karim SA, Naidoo K, Grobler A, et al. Initiating ART during TB treatment significantly increases survival: results of a randomized controlled clinical trial in TB/HIV-co-infected patients in South Africa. 16th Conference on Retroviruses and Opportunistic Infections, Montreal, QC, Canada, February 2009, abstract 36a. Available from www.retroconference.org/2009/Abstracts/34255. $\mathrm{htm}$ Date last accessed: April 14, 2009. Date last updated: February 12, 2009.

6 Breen RAM, Smith CJ, Bettinson $\mathrm{H}$, et al. Paradoxical reactions during tuberculosis treatment in patients with and without HIV co-infection. Thorax 2004; 59: 704-707.

7 Lawn SD, Wilkinson RJ, Lipman MC, et al. Immune reconstitution and "unmasking" of tuberculosis during antiretroviral therapy. Am J Respir Crit Care Med 2008; 177: 680-685.

8 Brastianos PK, Swanson JW, Torbenson M, et al. Tuberculosisassociated haemophagocytic syndrome. Lancet Infect Dis 2006; 6: 447-454.

9 Manabe YC, Breen R, Perti T, et al. Unmasked tuberculosis immune reconstitution inflammatory disease: a disease spectrum after initiation of antiretroviral therapy. J Infect Dis 2009; 199: 437-444.

10 Lehmann C, Wyen C, Hoffmann C, et al. Successful administration of aggressive chemotherapy concomitant to tuberculostatic and highly active antiretroviral therapy in a patient with AIDS-related Burkitt's lymphoma. HIV Med 2005; 6: 51-53. 